\title{
Assessment of Fiber-Enriched Pita Bread in The Lebanese Market: A Positive Correlation Between Dry Crude Fiber Content and TTA
}

\author{
Ali Al Khatib ${ }^{1}$, Hassan Hajj Hussein ${ }^{2}$, Zeinab Joumaa ${ }^{1}$, Mahmoud Farhat ${ }^{1}$, \\ Sami Tlais ${ }^{2 *}$
}

\begin{abstract}
${ }^{1}$ Nutrition and Food Science Department, Lebanese International University, Beirut, Lebanon.
${ }^{2}$ Biological and Chemical Department, Lebanese International University, Rayak, Lebanon.

*Corresponding Author
\end{abstract}

\begin{abstract}
Due to increased health awareness, many people are making the switch from the traditional low-fiber Lebanese white pita bread to healthier high-fiber pita bread alternatives. Even though the priceto-weight ratio of white pita bread is controlled and unified among all Lebanese bakeries and brands and is enforced by the Lebanese Ministry of Economy and Trade, there is a highly significant difference in the price-to-weight ratio among the different brands that offer high-fiber pita bread. We sought to investigate the root cause of this difference by assessing the composition of two of the most sold high-fiber pita bread types in Lebanon (brown and oat). We tested brown and oat pita bread from three different Lebanese bakeries for price-to-weight ratio, fiber content, TTA, $p H$, moisture, and calories per gram. Using Pearson correlation coefficient, we did not detect any correlation between the price and any of the tested properties; however, a highly positive correlation between crude fiber content and TTA was detected.
\end{abstract}

Keywords—Pita, bread, Fiber, TTA, bran, oat.

\section{INTRODUCTION}

Bread is the corner stone of the Lebanese diet; it is the essential ingredient of every meal consumed by the Lebanese public from breakfast to dinner, including snacks [1]. Traditionally, Pita bread was, and still is, used to scoop up food from the plate similar to using chips or crackers to dip into sauce, except that the deformability of pita bread allows for a more utilitarian use to grab solid or semi-solid food and to scoop up liquid or semi-liquid food [1]. Bread is also used to prepare wrap sandwiches in restaurants, for kids to take to school, and as quick snacks for the whole family.

In recent years, the global spread of fast food chains and the continued shift from a Mediterranean based diet to a more Westernized diet has led to a significant increase in obesity, high cholesterol, high blood pressure, diabetes, cognitive disorders, and other diseases among the Lebanese and Mediterranean populations [2]. In the wake of such transformations, many calls have been made by nutritionists and dieticians for a more balanced and healthy diet, which encouraged a large section of the Lebanese population to switch from white pita bread to a healthier alternative form of pita bread that contains more fiber (e.g. brown and oat) [1]. This shift in consumer demand has led to a shift in the baking industry towards a "healthy and fit" trend that has manifested as a clear diversification in the contents of the bread menu, which is constantly adding new varieties of healthier pita bread (low salt, low calories, high-fiber, fortified, etc.) [3]. Because almost everyone in Lebanon relies so heavily on bread in their diet, the Ministry of Economy and Trade (MOET) has long since taken it upon itself to control the price of wheat in order to make sure that the price of white pita bread remains affordable to all families, especially those with limited income. Unlike the case of white pita bread, which has a fixed price of $1.67 \mathrm{LL} / \mathrm{g}$, there is no control on the price of alternative bread, which allowed bakeries to set the price based on market demand, customer demographics, and perceived brand name.

In this study, we chose two of the most popular healthier alternatives to white pita bread (brown and oat), and especially since we could not find any published literature containing any assessments done on either type of bread in Lebanon. The Lebanese Standards Institution, LIBNOR, 
has a set of criteria for brown bread, which includes type of cereal used, type of yeast used, crude fiber content, moisture content, salt content, mineral oil content, and few others; however, it does not have any preset criteria for $\mathrm{pH}$ or TTA, both of which have a significant impact on bread freshness, taste, and microorganism growth [4]. In addition, LIBNOR has no clear criteria for oat bread. The assessment done in this study focused on the fiber content, moisture content, TTA, $\mathrm{pH}$, and calories in brown and oat pita bread purchased from three different bakeries that represent both multi-chain big businesses and small family owned and operated bakeries. Findings were compared with the adopted LIBNOR standards in terms of brown bread, and a comparison between brown and oat pita bread was done across the three different brands to determine if there are any major differences in the aforementioned properties that would explain or justify the significant difference in the price tag of these types of bread across the available brands.

\section{MATERIAL and METHODS}

\subsection{Samples}

Samples of bread were collected from 3 bakeries in Lebanon during Oct, 2019-Feb, 2020 period of time (major, medium and corner bakery). Two types of bread (Brown and Oat) were bought with three different production dates. Three samples of the two bread types were collected and coded as following: S1 brown, S2 oat, $\mathrm{W} 1$ brown, W2 oat, $\mathrm{C} 1$ brown, and $\mathrm{C} 2$ oat. Upon purchasing, the whole bread bag was weighed, and the loaves were counted. Each loaf was packed in a sealed bag. Samples were frozen at $-4^{\circ} \mathrm{C}$ until tested.

\subsection{Reagents}

All Chemicals were bought from Sigma-Aldrich.

\subsection{General Procedure for Crude Fiber Analysis}

The crude fiber was determined according to AOAC method (962.09) [5]. Two grams of $8 \mathrm{~h}$ dried bread (110 ${ }^{\circ} \mathrm{C}$ ) or none dried bread. The sample was extracted with $10.0 \mathrm{~mL}$ of dichloromethane. The remained solid was transferred to a $250 \mathrm{~mL}$ rounded flask, and $100 \mathrm{~mL}$ of 0.25 $\mathrm{N} \mathrm{H}_{2} \mathrm{SO}_{4}$ were added to the rounded flask with magnetic stirrer. After installation of reflux condenser, the flask was set under reflux in an oil bath for $30 \mathrm{~min}$. While hot, the solution was filtered through a Buchner funnel using a filter paper using vacuum filtration. The residue was washed with hot water until no longer acidic. The residue was then transferred to a rounded flask with addition of $100 \mathrm{~mL}$ of $0.3 \mathrm{M} \mathrm{NaOH}$ solution. The resulting slurry was refluxed for another $30 \mathrm{~min}$. While hot, the solution was filtered again using pre-weighted filter paper. The remaining crude fiber with filter paper was dried in the oven for $2 \mathrm{~h}$, and then it was cooled in the desiccator before reading its weight. The crude fiber content percentage was calculated by dividing the mass of crude fiber over the mass of sample [6].

\subsection{Moisture Content}

Moisture content of bread was determined by AOAC method (925.10) [7]. Two grams of bread sample was weighed and placed in a hot air oven for $8 \mathrm{~h}$ at $110{ }^{\circ} \mathrm{C}$. Samples were removed from the oven and cooled in a desiccator before measuring their weight. The percentage of moisture content was calculated based on the dried sample and the original sample [6].

$$
\text { 1.5. pH and TTA }
$$

pH and TTA (Titratable Acidity) of bread were determined by AACC 02-52.01 method. After mixing $10 \mathrm{~g}$ of bread sample with $90.0 \mathrm{~mL}$ of distilled water, the slurry was stirred for $30 \mathrm{~min}$. Then the $\mathrm{pH}$ was measured using calibrated pH meter (OHAUS STARTER300) [8].

\subsection{Calorimetry}

The samples were measured for their caloric content using an oxygen bomb calorimeter according to the manual provided with the calorimeter (IKA® ${ }^{\circledR} 200$, Werke $\mathrm{GmbH}$ \& Co.KG, Germany). All the samples were dried in an oven at $110^{\circ} \mathrm{C}$ for 8 hours

\subsection{Statistical Analysis}

All analyses were performed with three replicates. The statistical analyses were done using the IBM SPSS (Statistical Package for the Social Sciences, version 22.0) program. Analysis of variance consisted of univariate analysis was performed followed by Tukey's Honest Significant. Bivariate correlations obtained as Pearson's correlation coefficients (two tailed), cluster analysis, and principal component analysis were performed for analyzed bread samples using IBM SPSS.

\section{RESULTS}

1.8. Price

White pita bread is an essential part of the Lebanese diet, and its price-to-weight ratio is controlled and enforced by the Ministry of Economy and Trade (MOET) to make sure that every family in Lebanon can afford to put bread on the table. At the time when the samples were collected, the price for white pita bread was fixed at 1500 LL/900g (1.67 $\mathrm{LL} / \mathrm{g}$ ) regardless of the bakery or the region of sale. However, the MOET does not regulate the price-to-weight ratio of other types of pita bread (e.g. brown and oat). 
Both brown (designated by number 1) and oat (designated by number 2) pita bread were purchased from three different bakeries (designated by $\mathrm{C}, \mathrm{W}$, and $\mathrm{S}$ ). In our study, we weighed each pack of pita bread upon purchase and then used that value to determine the price to weight ratio for each of the 6 samples of bread used in the experiment. As expected, we detected a high significant difference $(p<0.001)$. In the price of the selected samples compared to white pita bread as the prices of brown and oat pita bread purchased from all three bakeries ranged between 2.26 and $8.84 \mathrm{LL} / \mathrm{g}$ (Fig. 1). In addition, when we compared the 6 different samples among themselves, only $\mathrm{W} 1$ and $\mathrm{S} 1$ were similar while all remaining products were significantly different from each other (Fig. 1). However, it was clear that the price of oat bread was significantly higher than the price of brown bread across all 3 bakeries (Fig. 1). In addition, brown and oat pita bread products of the $\mathrm{C}$ brand were significantly cheaper than brown and oat pita bread products of $\mathrm{S}$ and $\mathrm{W}$ brands, respectively, and the oat bread of the W brand (W2) stood out as the outlier of the group, nearly double the price of the nearest competitor (Fig. 1).

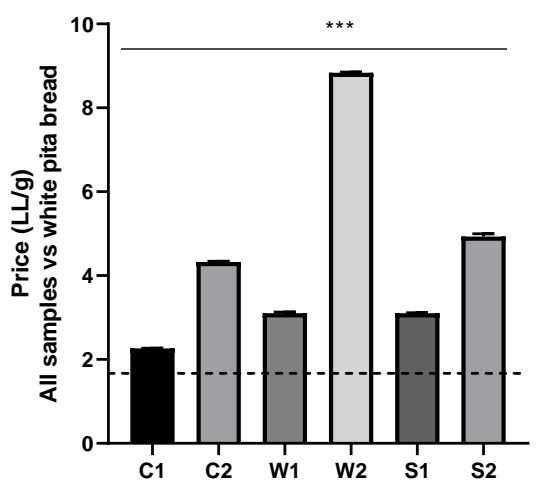

Fig.1: Price comparison among the six different samples of bread.

\subsection{Crude Fiber and Moisture Content}

Clearly both types of bread were priced differently and some brands were more expensive than others, however, was the difference due to a clear measurable difference in the composition of the bread, or were some bakeries selling their customers a product that was not compliant with the standards adopted by the Lebanese Standards Institution (LIBNOR)? To answer both questions, we conducted a series of experiments to assess the crude fiber and moisture content of the bread, in addition to several other properties.

According to LIBNOR standards, the crude fiber and moisture content of brown bread must not exceed 3 and $26 \%$, respectively; however, there are no stated regulations regarding the amount of soluble and insoluble dietary fibers in brown pita bread. In addition, there are no set standards for oat pita bread, which is why we compared the results of oat pita bread to the standards set for brown pita bread. Based on our findings, all tested samples had a dry crude fiber content that did not exceed the $3 \%$ limit, in fact, all samples had a crude fiber content that was significantly below the maximum allowed limit except for sample C2 (Fig. 2A). When looking into the moisture content, all samples were within the approved limit and only sample $\mathrm{C} 2$ was significantly below the maximum allowed limit (Fig. 2B).
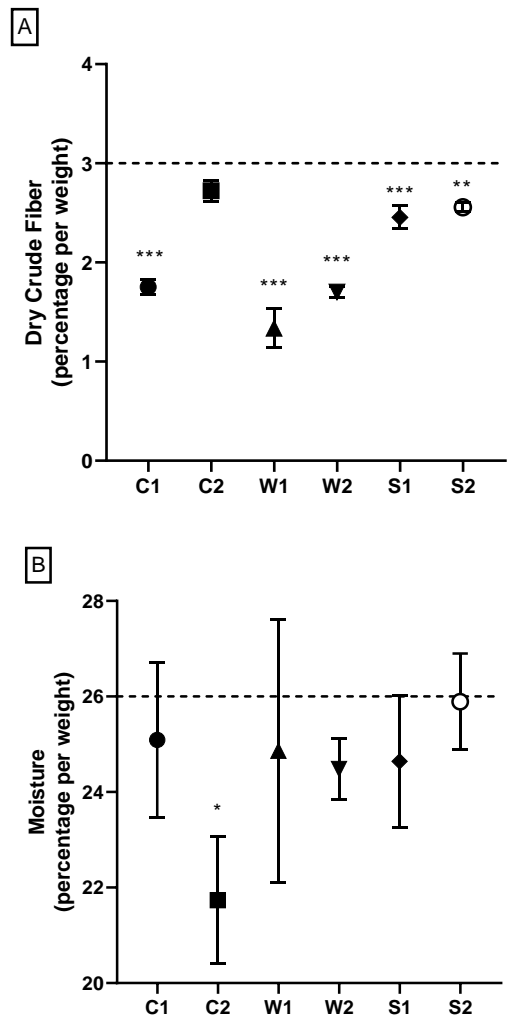

Fig.2: Dry crude fiber and moisture content of brown and oat pita bread.

\subsection{TTA, pH, and Energy}

We determined that all 6 bread samples were within the LIBNOR standards for crude fiber and moisture content, so we looked next at TTA, pH, and energy (Fig. 3). As was stated earlier, LIBNOR has no set standards for TTA, $\mathrm{pH}$, or energy for pita bread. Upon testing the samples, we did not detect any significant difference in the energy (cal/g) content among all 6 samples (Fig. 3C), in addition, only $\mathrm{C} 2$ and $\mathrm{W} 1$ exhibited a significant difference in $\mathrm{pH}$ when compared to each other $(p=0.0203$; Fig. 3B). However, comparing TTA values among all 6 samples showed a large margin of difference among the tested samples, but the statistical significance was not reported in figure $3 \mathrm{~A}$, instead, we reported the differences in TTA 
among all samples in the form of a comparison between each sample and all remaining samples, and we reported the results in figure 4 .
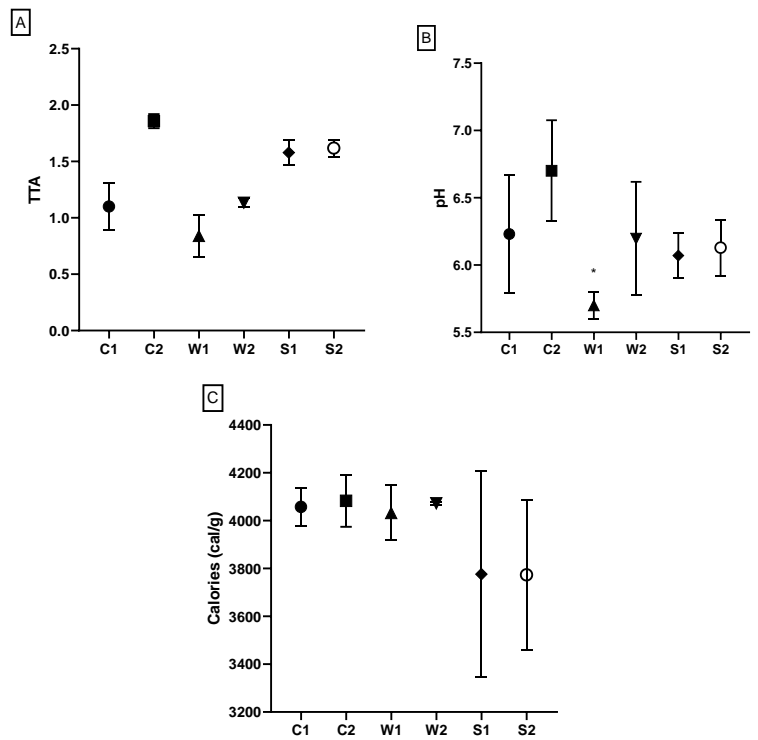

Fig.3: TTA, pH, and energy of brown and oat pita bread.

\subsection{Crude Fiber and TTA}

Initially, we determined that all tested samples were within the allowed range for crude fiber content, but since the difference in the price among all 6 samples was very highly significant (Fig. 1), we compared the crude fiber content and TTA among all 6 samples trying to determine if the differences in the price were correlated with the differences in crude fiber content and TTA values. Based on the literature, dietary fiber decreases the risk of colon cancer, optimizes blood lipids which helps in reducing the possibility of obesity, reduces hypertension and cardiovascular diseases, reduces the risk of developing diabetes in pre-diabetic people, and is a natural laxative that helps regulate bowl movement [9]. On the other hand, $\mathrm{pH}$ and TTA have a significant impact on bread quality. Both, $\mathrm{pH}$ and TTA, are dependent on fermentation time, sugar content, flour type, and other additives [10]. Generally speaking, the higher the TTA the more flavor the bread will have; however, very high levels can cause a sour off-flavor [11]; and since our initial results did not detect a significant difference in the $\mathrm{pH}$ level among the samples, we checked the TTA values only. We compared the crude fiber content of all 6 samples among each other, and aside from samples $\mathrm{C} 1$ and $\mathrm{W} 1$, all samples had significantly different amounts of crude fiber compared to each other (Fig. 4A-F). Also, we compared the TTA values for all 6 samples among each other and determined that $\mathrm{C} 1, \mathrm{~W} 1$, and $\mathrm{W} 2$ had similar TTA values were significantly lower than those for $\mathrm{C} 2, \mathrm{~S} 1$, and S2 (Fig. 4GL).
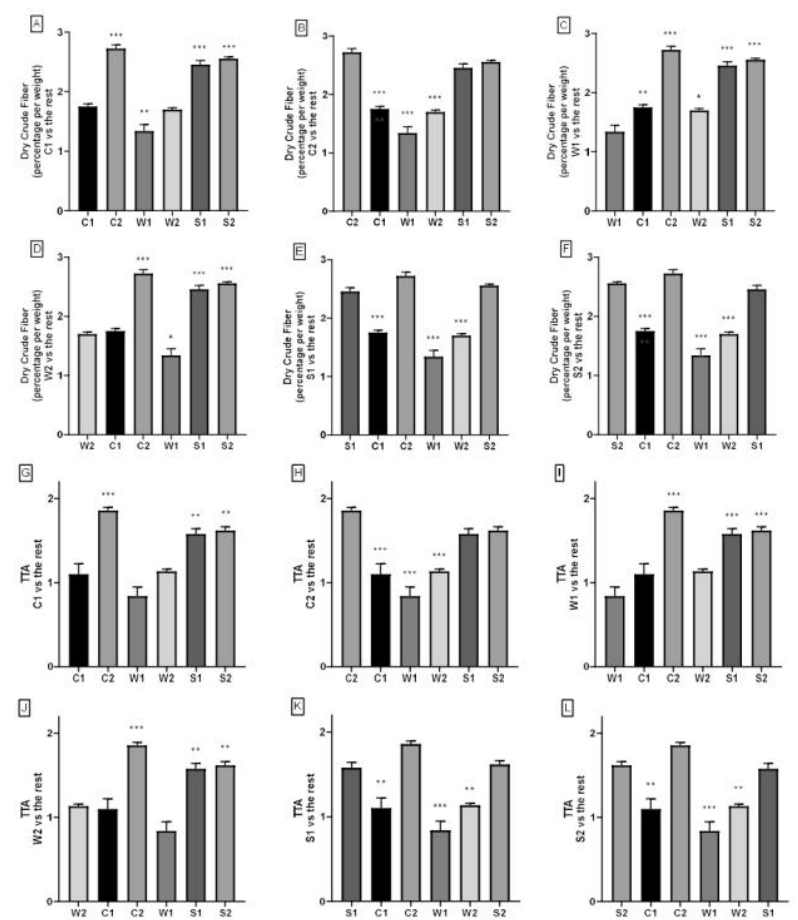

Fig.4: Crude fiber and TTA comparison among the six different samples of bread.

\subsection{Correlation between Crude Fiber and TTA}

Unfortunately, we were unable to find any correlation between the price of pita bread and any of the tested properties including crude fiber content and TTA values; however, a very interesting observation was the detection of a positive correlation between crude fiber content and TTA among the samples. A Pearson's $r$ was computed to assess the relationship between the amount of crude fiber found in the sample and the TTA value of the sample. We detected a positive correlation between the two variables, $r$ $=.959, \mathrm{n}=6, \mathrm{p}=.0025$, two-tailed. A scatterplot summarizes the results (Fig. 5).

\section{CONCLUSION}

As was mentioned earlier, pita bread is a staple food of the Lebanese diet, which is why the MOET regulates and enforces the price of wheat and flour that are sold to bakeries in order to fix the price of white pita bread that is sold to the public. However, people are increasingly switching from the traditional white pita bread to healthier forms of pita bread that contain more fiber, which bakeries still produce using the same wheat and flour that they 
purchase for a regulated price while adding few additives to the mix [12].

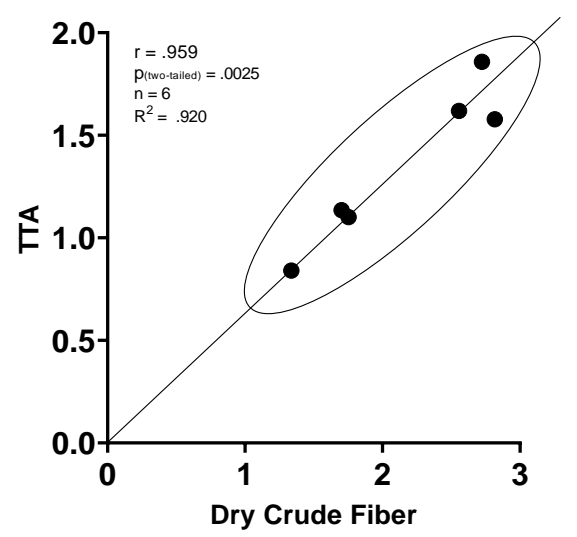

Fig.5: A scatterplot showing the correlation between TTA and Dry Crude Fiber content.

We determined that the price-to-weight ratios of brown and oat pita bread purchased from three different bakeries were significantly larger than that of white pita bread, which could be argued as being the cost of the other additives like bran, oats, etc. that bakeries use to make their high-fiber bread; however, even the ratios were significantly different among the same type of bread when compared across the three different brands. While trying to determine any possible differences among the different samples of bread that would justify the differences in price, we looked into crude fiber content, moisture, $\mathrm{pH}$, TTA, and energy.

The primary reason people are switching to alternative pita bread products is that they are seeking to increase the fiber intake in their diet. Increased dietary fiber intake helps decrease the risk of colon cancer, optimizes blood lipids which helps reduce the risk of obesity, hypertension, and cardiovascular diseases, and decreases D-glucose absorption and insulin secretion, which is very important in the prevention and control of diabetes. Also, fiber is a laxative, which helps regulate bowl movement [13]. Since LIBNOR has a set standard for crude fiber content for brown bread not to exceed $3 \%$, we compared the crude fiber content of all six samples of bread to determine if the fiber content was the primary reason for the difference in the price (i.e. more fiber equals higher price). Even though the tested samples had varying concentrations of crude fiber, they were all within the limit set by LIBNOR, but we did not detect any correlation between the price of the bread and its crude fiber content.

Moisture in bread has many implications on its hardness, chewiness, textural characteristics, taste, bacterial and mold growth, and shelf life [14]. When we tested the moisture content of all bread samples, we determined that all samples were within the limits set forth by LIBNOR (not to exceed 26\%); also, we did not detect any difference in the moisture content among the tested samples.

The $\mathrm{pH}$ and TTA values are significant factors that affect bread freshness, flavor, and microbiota. Both, $\mathrm{pH}$ and TTA, are dependent on fermentation time, yeast type, sugar content, flour type, and other additives [10]. The $\mathrm{pH}$ range for brown bread is usually between 2.5 and 7.0 depending on the strain of yeast used and duration of dough fermentation; while the TTA range for brown bread is usually between 0.6 and 4.25 , also depending on the strain of yeast used and duration of fermentation [15]. LIBNOR has no set standards for $\mathrm{pH}$ or TTA for brown or pita bread; however, the $\mathrm{pH}$ range of the tested samples was between 5.70-6.70 and the TTA range was between 0.84-1.86. Even though we did not detect major significant variations in the $\mathrm{pH}$ levels among the six bread samples, we did detect significant differences in the TTA levels. In fact, the six bread samples were divided into two groups in terms of TTA values where $\mathrm{C} 1, \mathrm{~W} 1$, and $\mathrm{W} 2$ had similar TTA values that were significantly lower than $\mathrm{C} 2, \mathrm{~S} 1$, and S2, which were similar among themselves. Similar to crude fiber and moisture, we were unable to detect any correlation between $\mathrm{pH}$ or TTA and the price of bread.

Finally, we looked into the energy content of the bread samples trying to determine if the price was related in a way to the caloric energy of the product (i.e. low-calories equal higher price). LIBNOR has no set values for the energy content of pita bread, and when we assessed the energy content of all samples, we determined that the samples ranged between 3773 and 4083 calories/gram with no significant difference among the tested samples. It was also surprising that two of the brands had the exact same nutritional food label. Such discrepancies in the label should be investigated by the Consumer Protection Agency.

At the end of our investigation, we were unable to detect any correlation between the price of the bread and any of the tested properties. It maybe some other property that we did not test (e.g. freshness, taste, elasticity, appearance, crumb color, crumb firmness, etc.), or it could be a matter of brand name perception. However, an interesting observation that was not reported by any previous study was the discovery of a very positive correlation between crude fiber content and TTA values. This finding is in line with a previous study that investigated the effect of the addition of live yeast cells on in vitro oat straw rumen fermentation, it was determined that live yeast cells reduced the $\mathrm{pH}$ of rumen by increasing the production of propionic acid, butyric acid, valeric acid, and isovaleric 
acid [16]. Further analysis on the correlation between TTA and crude fiber content should be carried out on several cereal products to determine if the simple and cheaper TTA test could be an accurate alternative to predict crude fiber content in cereal products such as bread and animal feed.

\section{REFERENCES}

[1] Chbeir R., Mikhael M. (2017). Breaking the Bread Market: Lebanon's Modern-day Bakeries. BLOMINVEST BANK S.A.L.; https://blog.blominvestbank.com [accessed date: Sept 22, 2020]

[2] Kopp W. (2019). How western diet and lifestyle drive the pandemic of obesity and civilization diseases. Diabetes, Metabolic Syndrome and Obesity: Targets and Therapy, 12: 2221-2236. Doi: 10.2147/DMSO.S216791

[3] Ibrahim U. K., Salleh R. M., and Maqsood-ul-Haque S. N. S. (2015). Bread towards Functional Food: An Overview. International Journal of Food Engineering. 1(1), 39-43; Doi: 10.18178/ijfe.1.1.39-43

[4] Tarar O.M., Ur-Rahman S., Ud Din G.M., Murtaza, M.A. (2010). Studies on the shelf life of bread using acidulants and their salts, Turkish Journal of Biology, 34, 133-138

[5] AOAC, 1990. AOAC Method 962.09 Official Methods of the Association of Official Analytical Chemists, 15th Edn., 777, Arlington, VA, USA

[6] Tiimub B. M. (2013). Proximate analyses of three brands of bread under different storage conditions available on the Ghanaian market. Food Science and Quality Management, $12,23-30$.

[7] AOAC, 1990. AOAC Method 925.10 Official Methods of the Association of Official Analytical Chemists, 15th Edn., 777, Arlington, VA, USA

[8] Yin Y.; Wang J.; Yang S.; Feng J.; Jia F.; Zhang C. (2015) Protein Degradation in Wheat Sourdough Fermentation with Lactobacillus plantarum M616. Interdisciplinary Sciences: Computational Life Sciences, 7, 205-210; Doi: 10.1007/s12539-015-0262-0

[9] Olubunmi I. P., Babatunde K. S., Bolanle O. O., Seyioba S. O., Taiwo L.T., Olukayode O. A., Nwankego E. G. (2015). Quality evaluation of fibre-enriched bread. International Journal of Nutrition and Food Sciences. 4(4), 503-508; Doi: 10.11648/j.ijnfs.20150404.23.

[10] Abedfar A., Sadeghi A. (2019). Response surface methodology for investigating the effects of sourdough fermentation conditions on Iranian cup bread properties. Heliyon. $\quad 5, \quad$ e02608; DOI: doi.org/10.1016/j.heliyon.2019.e02608

[11] Measuring $\mathrm{pH}$ and TTA, Baking Update, Practical technology from Lallemand Inc, (2018), volume 3; https://lallemandbaking.com/wp-
content/uploads/2018/04/3_15PREFERMENTS.pdf

[accessed date: Sept 22, 2020]

[12] Mounayar R., Jreij R., Hachem J., Abboud F. and Tueni M. (2019). Breakfast intake and factors associated with adherence to the Mediterranean diet among Lebanese high school adolescents. Journal of Nutrition and Metabolism, 2019, pp.1-10; Doi:10.1155/2019/2714286

[13] Kurek M., and Wyrwisz J. (2015). The Application of Dietary Fiber in Bread Products. Journal of Food Processing \& Technology. 6(5), 1-4, doi:10.4172/21577110.1000447.

[14] Pastuszka D., Gambuś H., Ziobro R., Mickowska B., Buksa K., Saba R. (2012). Quality and nutritional value of wheat bread with a preparation of oat proteins. Journal of Microbiology, Biotechnology and Food Sciences. 1(February Special issue), 980-987.

[15] Anastasio M., Pepe O., Cirillo T. et al. 2010). Selection and use of phytate-degrading lab to improve cereal-based products by mineral solubilization during dough fermentation. Journal of Food Science. 75. M28-35; Doi:10.1111/j.1750-3841.2009.01402.x

[16] Ruiz, O., Castillo, Y., Arzola, C., Burrola, E., Salinas, J., Corral, A., Hume, M. E., Murillo, M. And Itza, M. (2016). Effects of Candida norvegensis Live Cells on In vitro Oat Straw Rumen Fermentation. Asian-Australasian Journal of Animal Sciences, 29, 211-218; DOI: 10.5713/ajas.15.0166 